DrAfT VERSiOn June 22, 2021

Typeset using $\mathrm{LAT}_{\mathrm{E}} \mathrm{X}$ default style in AASTeX61

\title{
TOWARD A MEASUREMENT OF THE TRANSVERSE PECULIAR VELOCITY OF GALAXY PAIRS
}

\author{
Alexandra E. Truebenbach ${ }^{1}$ And Jeremy Darling ${ }^{1}$
}

${ }^{1}$ Center for Astrophysics and Space Astronomy,
Department of Astrophysical and Planetary Sciences,
University of Colorado, 389 UCB, Boulder, CO 80309-0389, USA

\section{ABSTRACT}

The transverse peculiar velocities caused by the mass distribution of large-scale structure provide a test of the theoretical matter power spectrum and the cosmological parameters that contribute to its shape. Typically, the matter density distribution of the nearby Universe is measured through redshift or line-of-sight peculiar velocity surveys. However, both methods require model-dependent distance measures to place the galaxies or to differentiate peculiar velocity from the Hubble expansion. In this paper, we use the correlated proper motions of galaxy pairs from the VLBA Extragalactic Proper Motion Catalog to place limits on the transverse peculiar velocity of galaxy pairs with comoving separations $<1500 \mathrm{Mpc}$ without a reliance on precise distance measurements. The relative proper motions of galaxy pairs across the line of sight can be directly translated into relative peculiar velocities because no proper motion will occur in a homogeneous expansion. We place a $3 \sigma$ limit on the relative proper motion of pairs with comoving separations $<100 \mathrm{Mpc}$ of $-17.4 \mu \mathrm{as} \mathrm{yr}^{-1}<\dot{\theta} / \sin \theta<19.8 \mu \mathrm{as} \mathrm{yr}^{-1}$. We also confirm that large-separation objects $(>200 \mathrm{Mpc})$ are consistent with pure Hubble expansion to within $\sim 5.3 \mu \mathrm{as} \mathrm{yr}^{-1}(1 \sigma)$. Finally, we predict that Gaia end-of-mission proper motions will be able to significantly detect the mass distribution of large-scale structure on length scales $<25 \mathrm{Mpc}$. This future detection will allow a test of the shape of the theoretical mass power spectrum without a reliance on precise distance measurements.

Keywords: proper motions — cosmology: large-scale structure of universe — astrometry — catalogs - quasars: general - cosmology: cosmological parameters 


\section{INTRODUCTION}

The modern standard model of cosmology is a spatially flat, expanding Universe dominated by cold dark matter and a cosmological constant. Small matter density perturbations in the primordial Universe drive the initial gravitational collapse of matter and dark matter into overdensities, which give rise to the formation of large-scale structure (LSS), clusters, and galaxies (e.g., Blumenthal et al. 1984) through a hierarchical process. The overall process and cosmological parameters that govern the process are, in general, well constrained (e.g., Hu \& White 1996a,b; Hu et al. 1997; Riess et al. 2001; Peiris et al. 2003; Moodley et al. 2004; Planck Collaboration et al. 2016a). However, the exact values of the parameters remain somewhat uncertain, including the baryon and neutrino density (e.g., Eisenstein \& Hu 1999; Eisenstein et al. 2005), the Hubble constant (e.g., Eisenstein et al. 2005; Riess et al. 2011; Planck Collaboration et al. 2016b; Riess et al. 2016; Zhang et al. 2017; Riess et al. 2018), the tensor-to-scalar ratio of primordial gravitational waves (e.g., Planck Collaboration et al. 2016c), and the spatial curvature (e.g., Eisenstein et al. 2005; Planck Collaboration et al. 2016d).

The current distribution of LSS provides a means to test many of the less certain cosmological parameters by comparing the observed LSS to that predicted by cosmological simulations. Most commonly, maps of LSS are created using the sky distribution of visible galaxies with redshift as a proxy for distance (e.g., de Lapparent et al. 1986; York et al. 2000; Gott et al. 2005). However, these maps rely on the assumption that light and visible matter trace the overall dark matter distribution. In general, it is reasonable to assume that galaxies form at the peaks of dark matter (Kaiser 1984), but a bias model (e.g., Bardeen et al. 1986; Coles 1993; Fry 1996; Tegmark \& Peebles 1998) is still required to translate the observed LSS to the dark matter distributions generated by cosmological simulations.

Galaxy line-of-sight peculiar velocities are an alternate means to track the dark matter distribution that does not require a translation between light and total mass because peculiar velocities of galaxies are directly caused by the matter density distribution. Line-of-sight peculiar velocities are obtained from the difference between the redshift and a redshift-independent distance. Velocity surveys can probe more distant structures than redshift surveys because objects' velocities can be influenced by distant objects that are outside the range of the survey (e.g., Doumler et al. 2013; Tully et al. 2014). The drawback of line-of-sight velocity surveys to detect the dark matter distribution is that small uncertainties in a galaxy's distance can translate to large peculiar velocity uncertainties. Therefore, large samples of galaxy redshifts and distances are needed to statistically detect an average matter density distribution (Tully et al. 2014).

Both methods of mapping LSS produce similar matter density distributions (e.g., Strauss et al. 1992; Dekel et al. 1993; Kitaura et al. 2012; Courtois et al. 2012), which is a good confirmation of the standard model of LSS evolution through hierarchical growth and gravitational instability. However, both methods require redshifts or other modeldependent distance measures to either spatially place the galaxies or to translate spectroscopic line shifts into peculiar velocities. Therefore, another method that is independent of the "distance ladder" and other distance models is needed to provide an independent test of the model of LSS evolution.

Extragalactic proper motions can be used to test models of LSS evolution without a reliance on the "distance ladder." Like line-of-sight peculiar velocities, transverse peculiar velocities directly probe the matter density distribution and do not require any assumptions about the relative abundances of visible matter and dark matter (or how light traces mass). As the Universe expands, both line-of-sight and peculiar velocities will contain peculiar motion from gravitational interactions. However, line-of-sight velocities require an independent distance measure to differentiate between Hubble expansion and peculiar velocity. On the other hand, orthogonal velocities across the line of sight (observed as proper motions) are separable from the Hubble expansion because no proper motion will occur in a homogeneous expansion (Nusser et al. 2012; Darling 2013).

In this paper, we use the relative proper motion of pairs of extragalactic objects from our VLBA Extragalactic Proper Motion Catalog (Truebenbach \& Darling 2017) to constrain transverse peculiar velocities induced by the mass distribution of LSS. We describe the expected signal in Section 2 and calculate the signal for pairs with separations $<1500 \mathrm{Mpc}$ is Section 3. In Section 4, we compare our measurement to that predicted by a transverse peculiar velocity two-point correlation statistic calculated from a $z=0$ matter power spectrum (Darling \& Truebenbach 2018 submitted). Finally, we predict Gaia's ability to measure the relative convergence of galaxies on small scales (Sec. 5) and discuss future improvements to our catalog (Sec. 6). In this paper we assume $H_{0}=70 \mathrm{~km} \mathrm{~s}^{-1} \mathrm{Mpc}^{-1}$ and a flat cosmology with $\Omega_{\Lambda}=0.73$ and $\Omega_{M}=0.27$.

\section{PAIRWISE PROPER MOTION - THEORY}


Extragalactic proper motions are a combination of intrinsic apparent proper motions that are specific to each individual source and uncorrelated between objects (e.g., radio jets), correlated proper motions from cosmological effects (e.g., Gwinn et al. 1997; Quercellini et al. 2009; Nusser et al. 2012; Darling 2013), and apparent proper motions from observer-induced signatures such as the secular aberration drift (e.g., Fanselow 1983; Bastian 1995; Eubanks et al. 1995; Sovers et al. 1998; Mignard 2002; Kovalevsky 2003; Kopeikin \& Makarov 2006; Titov et al. 2011; Titov \& Lambert 2013; Xu et al. 2012, 2013). Extragalactic proper motions measured with very long baseline interferometry (VLBI) at

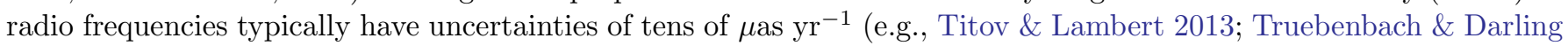
2017) that are much larger than the predicted cosmological proper motions $\left(\lesssim 15 \mu \mathrm{as} \mathrm{yr}^{-1}\right)$. Therefore, studies using extragalactic proper motions to measure correlated cosmological and observer-induced effects are challenged by randomly-oriented intrinsic apparent proper motions and proper motion uncertainties.

The majority of extragalactic proper motions are measured using VLBI observations of radio-loud quasars. However, in addition to proper motion caused by cosmological effects, quasars also have intrinsic proper motions, predominantly due to the motion of plasma in relativistic jets produced by the quasars (e.g., Bridle \& Perley 1984; Fey et al. 1997). These intrinsic proper motions are random in orientation on the sky. To separate intrinsic proper motions from cosmological proper motions, we use the relative proper motions of "pairs" of quasars (Darling 2013). The intrinsic proper motions of quasars are uncorrelated, whereas LSS growth will display a correlated signal in close-separation pairs along the pair axis. With a large enough sample size and a long enough observing period, the contaminating signal caused by intrinsic motions can be reduced enough to detect the correlated proper motion caused by the mass distribution of LSS.

For a "pair" of extragalactic objects ${ }^{1}$, the angular separation of the objects is defined as

$$
\sin \theta=\frac{l}{D_{A}}
$$

where $l$ is the proper length separating the objects and $D_{A}$ is the angular diameter distance. Then, the relative proper motion of the two objects along a great circle, $\dot{\theta}$, is

$$
\frac{\dot{\theta}}{\sin \theta}=\frac{-\dot{D_{A}}}{D_{A}}+\frac{i}{l}=\frac{-H(z)}{1+z}+\frac{i}{l}
$$

where

$$
H(z)=H_{0} \sqrt{\Omega_{M, 0}(1+z)^{3}+\Omega_{\Lambda}},
$$

and $i$ is the change in proper length (Darling 2013). See Darling (2013) for further discussion.

If the pair of extragalactic objects are far enough apart that they do not interact gravitationally, then

$$
\frac{i}{l}=\frac{H(z)}{1+z}
$$

because the space between the objects expands with the Hubble expansion. Combining this equation and Equation 2 gives $\dot{\theta}=0$ - no proper motion is expected for objects comoving with a homogeneously expanding Universe. On the other hand, if the pair are part of a static structure (e.g., two galaxies in the same virialized cluster) then $i / l=0$ and the pair will appear to converge as they move away from us with the Hubble flow. For a static structure at $z=0$, the

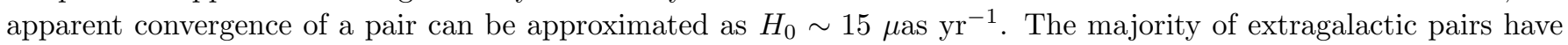
large physical separations and will show the null signal expected for gravitationally non-interacting objects entrained in the Hubble flow. However, pairs with close physical separations ( $\lesssim 50 \mathrm{Mpc}$, comoving) are expected to show relative proper motion that is a combination of the two signals; the pair will both appear to converge as the galaxies move away from us with the Hubble flow and they will have relative proper motion determined by their gravitational interaction with their local mass density distribution. Therefore, most pairs are expected to have a total relative proper motion that is less than the $\sim 15 \mu$ as $\mathrm{yr}^{-1}$ signal predicted for static structures.

\section{PAIRWISE PROPER MOTION - MEASUREMENT}

1 "Pair" signifies any two randomly selected objects and does not denote any association between the two objects. 
We measured the relative proper motion of extragalactic objects using the VLBA Extragalactic Proper Motion Catalog (Truebenbach \& Darling 2017). The catalog contains 713 proper motions measured with VLBI, 596 of which have well measured redshifts and are therefore usable for this measurement. Redshifts are required to calculate physical separations between pairs. We excluded all catalog objects with either no redshift or a flagged redshift in the VLBA Extragalactic Proper Motion Catalog. A flagged redshift indicates that the redshift is either photometric or uncertain.

Prior to calculating pairwise proper motions, we also fit and subtracted the secular aberration drift signal measured in our catalog (Truebenbach \& Darling 2017). The secular aberration drift, an apparent curl-free dipole motion of all extragalactic objects caused by the Solar System's acceleration towards the Galactic center, is an observer-induced apparent extragalactic motion and must be removed before analyzing any proper motions caused by cosmological effects. In Truebenbach \& Darling (2017), we measured a dipole signal from the aberration drift with a square root power equal to $4.89 \pm 0.77 \mu$ as $\mathrm{yr}^{-1}$ and an apex of $\left(275.2 \pm 10.0^{\circ},-29.4 \pm 8.8^{\circ}\right)$ in equatorial coordinates (see $\mathrm{Xu}$ et al. (2013) and Titov \& Lambert (2013) for other measurements of the secular aberration drift). Although we only detect a portion of the predicted dipole signal (expected square root power $=15.6 \pm 2.3 \mu \mathrm{as}^{-1}$; derived from Reid et al. 2009) in our catalog, we still must remove this partial dipole before proceeding.

For all objects, we calculated their relative proper motion, $\dot{\theta} / \sin \theta$, with respect to all other sources in the catalog. For two points on a sphere, the angular separation of the two points is

$$
\theta_{i j}=\arccos \left(\sin \delta_{i} \sin \delta_{j}+\cos \delta_{i} \cos \delta_{j} \cos \left[\alpha_{i}-\alpha_{j}\right]\right)
$$

where the points' equatorial coordinates are $\left(\alpha_{i}, \delta_{i}\right)$. The change in the angular separation due to the proper motion of those points is

$$
\begin{aligned}
\dot{\theta}_{i j}=-\left(\cos \delta_{i} \sin \delta_{j}\left[\mu_{\delta, i}-\mu_{\delta, j} \cos \left(\alpha_{i}-\alpha_{j}\right)\right]+\sin \delta_{i} \cos \delta_{j}\left[\mu_{\delta, j}-\mu_{\delta, i} \cos \left(\alpha_{i}-\alpha_{j}\right)\right]\right. \\
\left.-\cos \delta_{i} \cos \delta_{j} \sin \left(\alpha_{i}-\alpha_{j}\right)\left[\mu_{\alpha, i}-\mu_{\alpha, j}\right]\right) / \sin \theta_{i j}
\end{aligned}
$$

(Darling 2013).

Comoving separation is calculated from the angular separation and the cosine rule:

$$
D_{i j}^{2}=D_{i}^{2}+D_{j}^{2}-2 D_{i} D_{j} \cos \theta_{i j}
$$

$D_{i}$ and $D_{j}$ are the comoving proper distances of the objects, calculated by integrating the inverse of the scale factor from the object's time of emission to the present:

$$
D_{i}=c \int_{t_{i}}^{0} \frac{d t^{\prime}}{a\left(t^{\prime}\right)} .
$$

Several galaxies (M81, M84, 3C274, 1923+210, and NGC4261) are too nearby for redshift to be a good proxy for distance. For these, we used a median of various redshift-independent distances reported by the NASA/IPAC Extragalactic Database $^{2}$ (NED), including distances measured from Type 1a supernovae light curves (e.g., Colgate 1979; Riess et al. 1996; Perlmutter et al. 1999), globular cluster luminosity functions (e.g., Hanes 1977; Harris \& Racine 1979), surface brightness fluctuations (Tonry \& Schneider 1988; Tonry et al. 1990; Jensen et al. 1998; Blakeslee et al. 1999; Mei et al. 2005), and the Faber-Jackson relation (Faber \& Jackson 1976). We did not include distance measurements made prior to the year 2000.

Figure 3 shows the relative proper motion of radio source pairs binned by comoving separation. The relative proper motions and error bars are the median and standard deviation of a bootstrap distribution (see below). The solid line at $\dot{\theta} / \sin \theta=-15 \mu$ as $\mathrm{yr}^{-1}$ indicates the expected convergence of a static structure at $z=0$. This represents the most extreme deviation from the null signal of pure Hubble expansion for pairs whose relative proper motion is not dominated by intrinsic motions (i.e., radio jets). The curved lines show the predicted relative proper motion as a function of pair separation estimated as the square root of the transverse peculiar velocity two-point correlation function for a pair with an angular separation of 30 degrees (see Section 4 and Darling \& Truebenbach 2018 submitted). It is important to note that individual objects can be part of many pairs, therefore the bins in Figure 3 are not statistically independent.

\footnotetext{
${ }^{2}$ https://ned.ipac.caltech.edu
} 


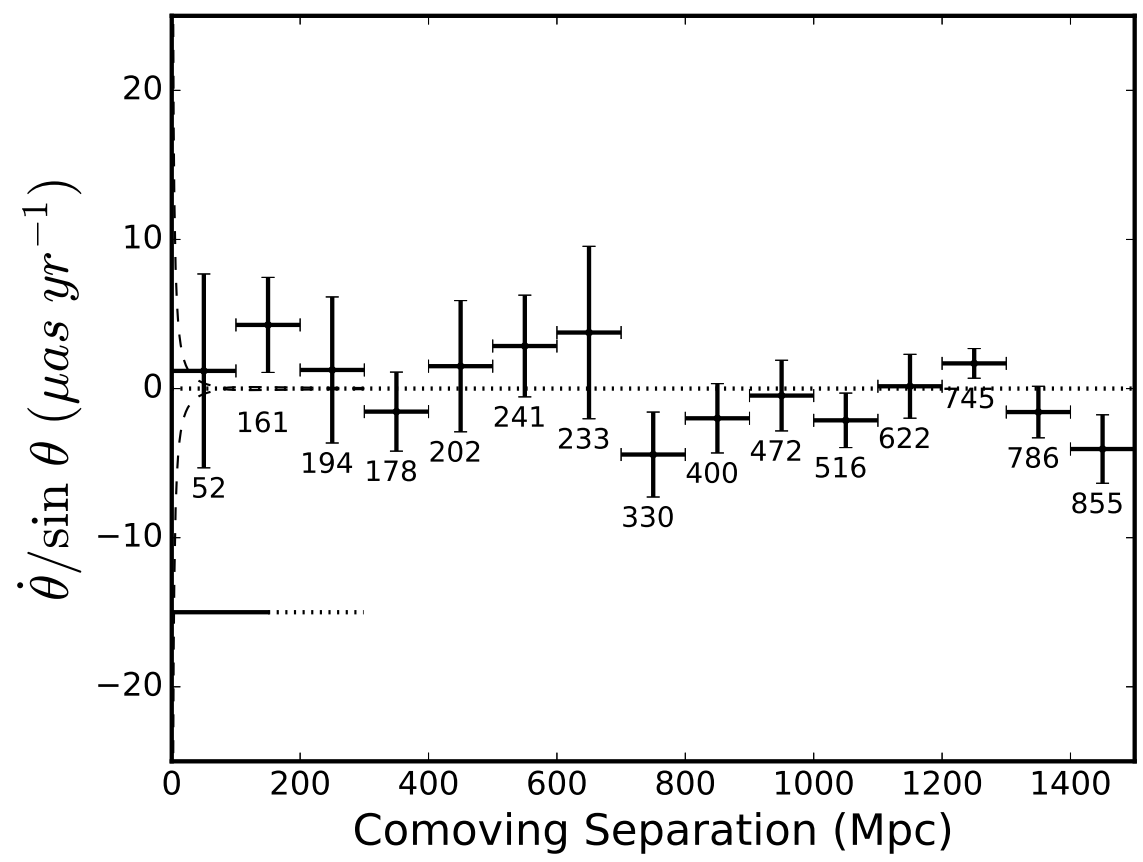

Figure 1. Measured pairwise divergence or convergence vs. comoving separation of radio sources in the proper motion catalog. Numbers beneath each data point indicate the number of pairs in each bin. The dotted line shows the null signal expected for gravitationally non-interacting pairs expanding with the Hubble flow, while the solid line at $\dot{\theta} / \sin \theta=-15 \mu$ as $\mathrm{yr}^{-1}$ indicates the expected convergence of a static structure at $z=0$. The dashed curved lines show the predicted relative proper motion of a pair with an angular separation of $30^{\circ}$ derived from the transverse, peculiar velocity two-point correlation function (Eqn 11 and Darling \& Truebenbach 2018 submitted). We find a 3 sigma limit on the convergence rate of gravitationally-interacting objects

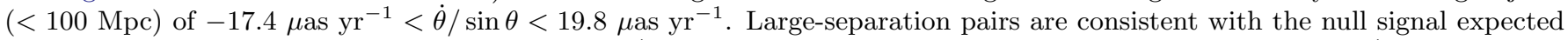

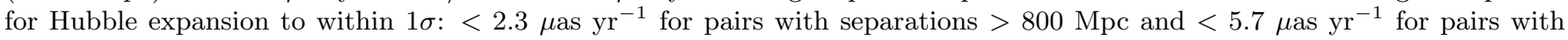
separations $200-800 \mathrm{Mpc}$.

Table 1 lists the bin medians and uncertainties. As predicted, bins with comoving separations $>100$ Mpc show no relative motion and are consistent with pure Hubble expansion. Despite the large scatter of relative proper motions within each bin due to the intrinsic proper motions from jets, we are still able to measure the expected cosmological behavior of large-separation pairs to high precision. Large-separation pairs are consistent with pure Hubble expansion

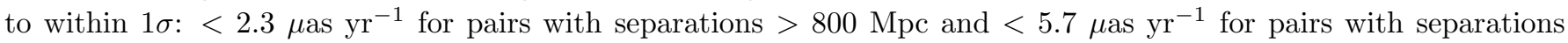
$200-800 \mathrm{Mpc}$. This result is a strong verification of our ability to place limits on small relative proper motion signals from a sample of large, uncorrelated intrinsic proper motions.

The bin with comoving separations $<100 \mathrm{Mpc}$ is consistent with both Hubble expansion and the maximum convergence of static structures at $z=0$. The predicted relative proper motion from the two-point correlation function lies between these two expected signals and is also consistent with the first bin. Overall, the $<100 \mathrm{Mpc}$ bin contains too few pairs to detect any significant deviation from pure Hubble expansion. Additionally, Figure 3 shows that the predicted two-point correlation function only deviates from zero for pairs with comoving separations $\lesssim 50 \mathrm{Mpc}$. There

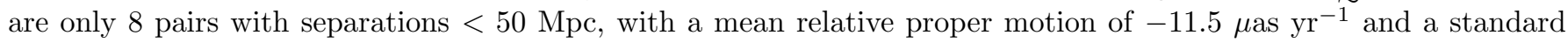
deviation of $22.2 \mu \mathrm{as} \mathrm{yr}^{-1}$. Therefore, there are too few pairs with separations $<50 \mathrm{Mpc}$ to statistically differentiate between pure Hubble expansion and the convergence expected for small-separation pairs.

The primary concern when calculating binned pairwise proper motion is the effect of individual objects with wellmeasured, large, intrinsic proper motions from relativistic jets. These intrinsic proper motions are often highly significant outliers in the bin and can dominate an error-weighted least-squares fit. This effect is particularly a concern for the bin of pairs with comoving separations $<100 \mathrm{Mpc}$. Figure 2 shows the objects that comprise the first bin along the outer circle, while the connecting chords show the pairs. This figure demonstrates that many of the pairs in the 
Table 1. Binned Pairwise Proper Motions

\begin{tabular}{lccc}
\hline \hline Comoving & $\langle z\rangle$ & $\langle\dot{\theta} / \sin \theta\rangle$ & $\mathrm{N}$ \\
Separation & & & \\
(Mpc) & & $\left(\mu \mathrm{as} \mathrm{yr}^{-1}\right)$ & \\
\hline $0-100$ & 0.10 & $1.2(6.2)$ & 52 \\
$100-200$ & 0.38 & $4.3(3.0)$ & 161 \\
$200-300$ & 0.21 & $1.3(5.0)$ & 194 \\
$300-400$ & 0.49 & $-1.7(4.2)$ & 178 \\
$400-500$ & 0.62 & $1.7(4.2)$ & 202 \\
$500-600$ & 0.57 & $2.6(3.3)$ & 241 \\
$600-700$ & 0.65 & $3.7(5.7)$ & 233 \\
$700-800$ & 0.67 & $-4.2(2.7)$ & 330 \\
$800-900$ & 0.77 & $-2.0(2.2)$ & 400 \\
$900-1000$ & 0.77 & $-0.9(2.3)$ & 472 \\
$1000-1100$ & 0.83 & $-2.2(1.7)$ & 516 \\
$1100-1200$ & 0.86 & $0.2(2.2)$ & 622 \\
$1200-1300$ & 0.89 & $1.7(0.9)$ & 745 \\
$1300-1400$ & 0.95 & $-1.3(1.7)$ & 786 \\
$1400-1500$ & 0.96 & $-4.0(2.3)$ & 855 \\
\hline
\end{tabular}

Note-Columns from left to right: Pair comoving separation, average redshift of pairs within separation range, median relative proper motion of the bootstrap distribution, and number of pairs within separation range. Parenthetical values indicate $1 \sigma$ standard deviation of the bootstrap distribution.

first bin are composed of the same small number of galaxies. If one galaxy that is in many pairs has a significant intrinsic proper motion, it can have a large effect on the bin's average.

We use relative proper motions along a pair axis to somewhat mitigate this effect. Intrinsic proper motions are randomly oriented and are uncorrelated between objects, reducing the likelihood that the intrinsic proper motion will lie along the pair axis. However, Figure 3 shows the individual pairwise proper motions for all pairs with comoving separations $<100 \mathrm{Mpc}$ and illustrates that the bin uncertainty is still dominated by significant individual pairwise proper motions. To quantify this scatter and reduce the influence of individual galaxies on the bin average, the bin averages and uncertainties in Figure 3 are the median and standard deviation of 1,000 iterations of a bootstrap distribution where subsets of the galaxies that comprise the bin are selected. In addition, the bin uncertainties must be bootstrapped because the bins are correlated - multiple bins contain many of the same objects. Therefore, we cannot directly calculate an uncertainty for each bin without also accounting for its correlation to the other bins. In this case, a bootstrap distribution is an accurate way to estimate the bin uncertainties.

We also reduce the influence of significant outliers by calculating the bin average of each bootstrap binning iteration through a maximum-likelihood "permissive fit" method (see Darling et al. 2018 submitted). This method allows for highly significant outlier data points by assuming that the offset between model and data will in some cases be bounded 


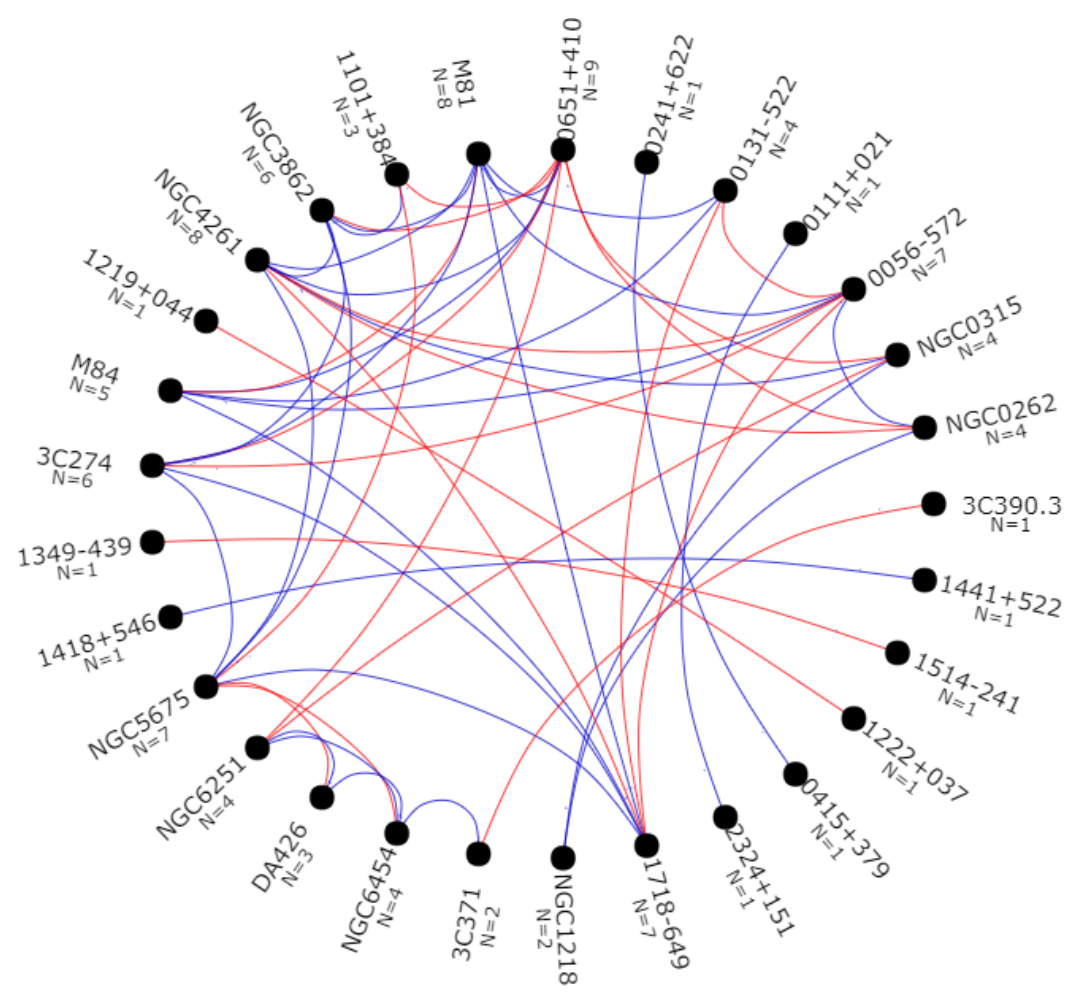

Figure 2. Chord diagram showing all objects that have pairs within $100 \mathrm{Mpc}$, comoving. The lines connect all objects that are pairs with each other. Blue lines indicate converging pairs $(\dot{\theta} / \theta<0)$, while red lines indicate diverging pairs $(\dot{\theta} / \theta>0)$. The number of pairs are also listed underneath each object. All pairs whose relative velocity is three times greater than the expected relative velocity of a static structure entrained in the Hubble flow (Eqn. 10) are removed from the plot.

by the measured uncertainty (Sivia \& Skilling 2006, p. 168). For each iteration, we use a least-squares technique with lmfit (Newville et al. 2014) to obtain the bin average that minimizes the permissive fit residual.

Before we bootstrap and fit each bin, we clip all pairs whose relative velocity is greater than three times that expected for a static structure at the pair's redshift. For a pair at an average comoving proper distance $D$, with relative apparent velocity $v=(\dot{\theta} / \sin \theta) D$, the maximum convergence expected for a static pair that is entrained in the Hubble flow is

$$
\frac{\dot{\theta}}{\sin \theta}=\frac{-H(z)}{1+z}
$$

which equates to a maximum expected relative velocity of

$$
v_{\max }=\frac{-H(z)}{1+z} D
$$

We include in our bin averages all pairs whose relative apparent velocity is within $1 \sigma$ of the range $3 v_{\max }<v<-3 v_{\max }$, thereby removing all pairs that are contracting / expanding more than three times faster than the contraction expected for a static structure receding in the Hubble flow. This clipping of large apparent relative velocities removes pairs whose relative proper motions are jet-dominated rather than dominated by peculiar velocities caused by the mass distribution of LSS. Figure 3 shows the clipped pairs from the first bin in red. This clipping criterion is preferable over simpler criteria (e.g., clipping objects with absolute proper motions or proper motion uncertainties $>100 \mu$ as $\mathrm{yr}^{-1}$ ) because it is distance independent. If we clip objects with large absolute proper motions and proper motion uncertainties, we remove the most distant objects from our bin averages because peculiar velocity uncertainty is higher for more distant objects; these criteria bias the bin averages towards nearby pairs. On the other hand, our criterion clips using a $1 \sigma$ deviation from the maximum expected relative velocity determined from the Hubble flow, which scales with distance. 


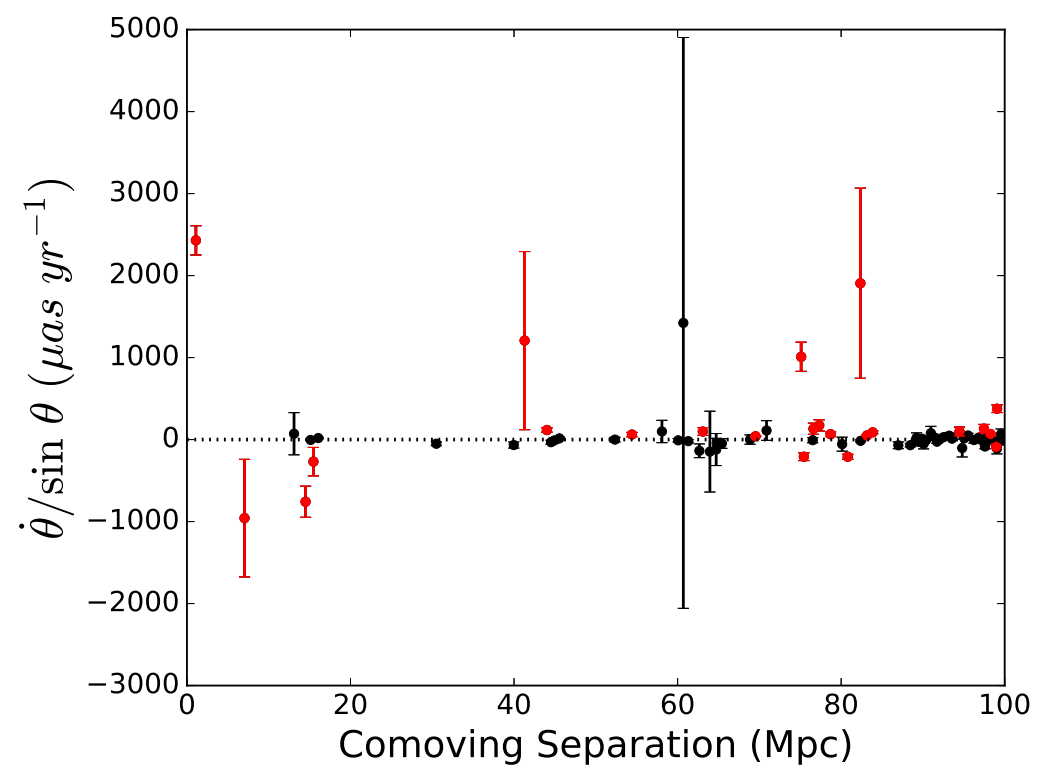

Figure 3. Measured pairwise divergence or convergence vs. comoving separation for all individual pairs with comoving separations < $100 \mathrm{Mpc}$. Black points are pairs included in the bin median, while red points are pairs that were clipped because their relative velocity is more than $1 \sigma$ greater than three times the expected relative velocity for a static structure at the pair redshift. The dashed line shows the null signal expected for large-separation, gravitationally non-interacting pairs expanding with the Hubble flow.

Finally, we include three rounds of $5 \sigma$ clipping of pairs that deviate from the bin median. On average, this iterative clipping removes $<1$ pair from each bootstrap iteration for bins with separations $<500 \mathrm{Mpc}$. It removes $1-6$ pairs for bins with separations $>500 \mathrm{Mpc}$.

\section{LIMIT ON PECULIAR TRANSVERSE VELOCITY}

As discussed in Section 3 and shown in Figure 3, the bin of pairs with comoving separations $<100 \mathrm{Mpc}$ is consistent with both pure Hubble expansion and the expected convergence of a static structure at $z=0$. It is also consistent with the predicted relative proper motion of a pair with an angular separation of $30^{\circ}$ derived from the transverse peculiar velocity two-point correlation function (Eqn 11). Based on the $<100 \mathrm{Mpc}$ bin median, we find a $3 \sigma$ constraint on the convergence rate of gravitationally-interacting objects of $-17.4 \mu \mathrm{as} \mathrm{yr}^{-1}<\dot{\theta} / \sin \theta<19.8 \mu$ as $\mathrm{yr}^{-1}$. Large-separation pairs are consistent with pure Hubble expansion to within $1 \sigma:<2.3 \mu \mathrm{as} \mathrm{yr}^{-1}$ for pairs with separations $>800 \mathrm{Mpc}$



The curved line representing the expected signal from the correlation function in Figure 3 is a rough approximation meant to aid in the reader's interpretation of the plot. The expected pair proper motion for close-separation pairs can be more precisely calculated using a two-point correlation statistic and a theoretical mass power spectrum. For a pair of objects with transverse peculiar velocities $\vec{v}_{\perp, 1}$ and $\vec{v}_{\perp, 2}$, respectively, Darling \& Truebenbach (2018 submitted) define a two-point correlation statistic,

$$
\xi_{v, \perp}\left(\vec{D}_{1}, \vec{D}_{2}\right)=\left\langle\left(\vec{v}_{\perp, 1} \cdot \hat{x}\right)\left(\vec{v}_{\perp, 2} \cdot \hat{x}\right)\right\rangle
$$

where the angle brackets represent summing over all pairs with a given separation, $\vec{D}_{1}$ and $\vec{D}_{2}$ are the radial vectors to the objects, and $\hat{x}$ is the unit space vector connecting the two radial vectors. In Appendix A, we calculate $\xi_{i}$, the transverse peculiar velocity two-point correlation statistic for each individual pair.

Figure 4 shows the median of the bootstrap distribution for the individual two-point correlation statistic (Eqn A7) for all pairs of objects with comoving separations $<1500 \mathrm{Mpc}$. The pairs are selected and clipped in the same manner as was used to create Figure 3. Again, the bin centers and error bars are the median and standard deviation of a 1000 iteration bootstrap distribution. The expected correlation statistic from the theoretical mass power spectrum is also plotted for several angular separations. Like Figure 3, this figure shows that the bin with comoving separations $<100$ $\mathrm{Mpc}$ is consistent with both pure Hubble expansion and the predicted relative motions of close-separation pairs from 


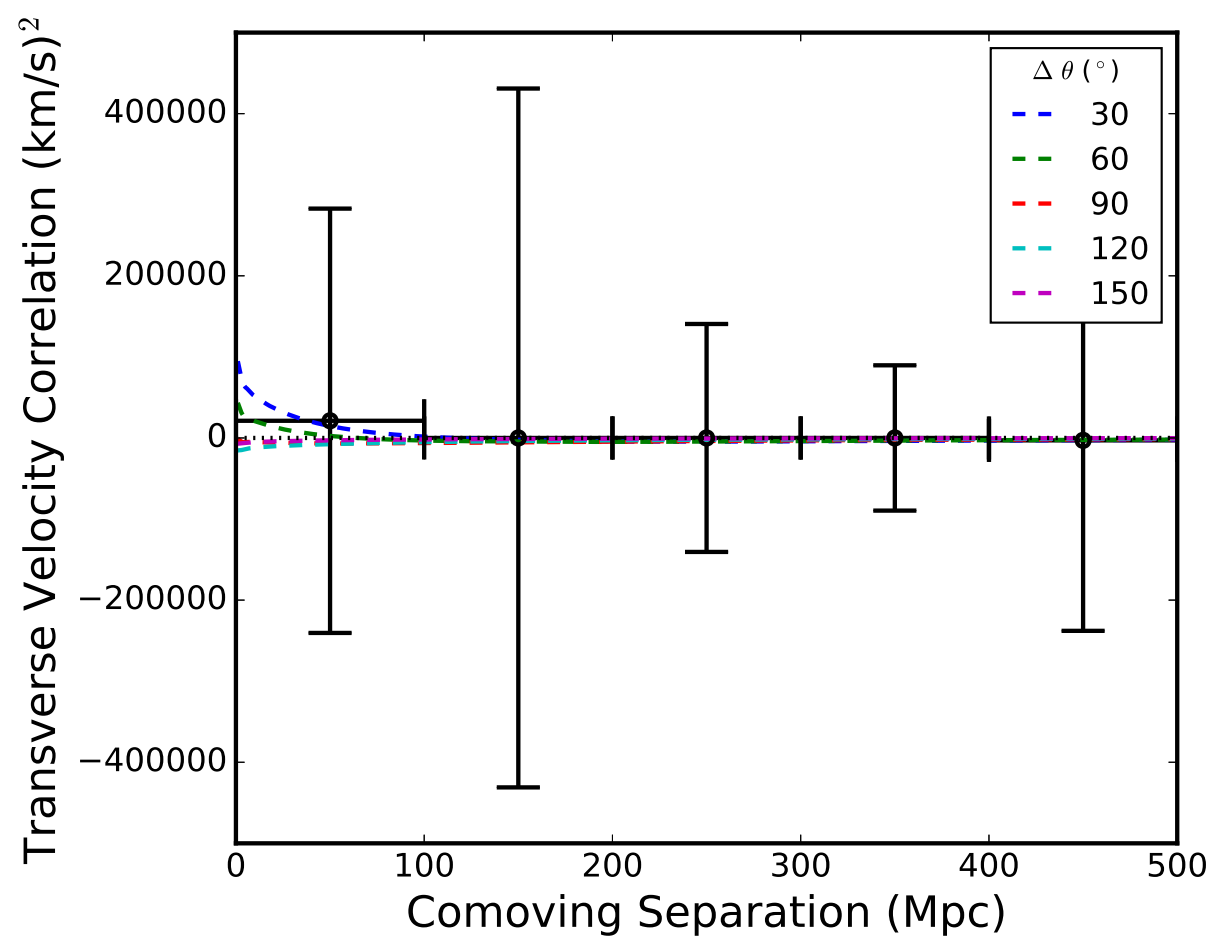

Figure 4. The average individual transverse peculiar velocity two-point correlation statistic (Eqn A7) for all pairs of objects with comoving separations $<500 \mathrm{Mpc}$. The expected correlation statistic from the theoretical mass power spectrum is plotted for several angular separations as dashed colored lines. The dotted black line is the expected null signal for pure Hubble expansion. The number of pairs per bin is the same as for Figure 3.

the mass power spectrum at several angular separations. The $<100 \mathrm{Mpc}$ bin contains a roughly even distribution of pair angular separations between $0^{\circ}$ and $180^{\circ}$ (most pairs in the bin include very nearby galaxies within 200 comoving Mpc of our galaxy). The plotted correlation statistics show that the expected $\xi_{i}$ vary widely as a function of angular separation, therefore the bin median is somewhat diluted by a wide range of $\xi_{i}$ values. In future studies with larger sample sizes, it may be beneficial to bin pairs by angular separation.

\section{FUTURE LIMITS ON PECULIAR TRANSVERSE VELOCITY WITH GAIA}

Our current catalog of extragalactic proper motions is not sufficiently large to detect the average motion caused by the mass distribution of LSS. Specifically, we lack sufficient pairs with comoving separations $<50 \mathrm{Mpc}$, where the predicted correlation of LSS deviates from the null signal predicted on large length scales. If we roughly expect the error bars in Figure 4 to decrease by $N$, then we would need $\sim 30$ more close-separation pairs to significantly detect the relative proper motion from LSS (this assumes that all new pairs are independent measurements and that multiple pairs do not contain the same object).

Gaia (Gaia Collaboration et al. 2016, 2018) extragalactic proper motions will significantly increase the sample of pairs with separations $<50 \mathrm{Mpc}$. However, Gaia proper motions have much higher uncertainties $\left(\sim 200 \mu \mathrm{as}_{\mathrm{yr}}^{-1}\right.$; Paine et al. 2018 submitted) than those produced through VLBI observations (10's of $\mu \mathrm{as} \mathrm{yr}^{-1}$; Titov \& Lambert 2013; Truebenbach \& Darling 2017). To assess the contribution of Gaia proper motions to our measurement of the peculiar velocities caused by the mass distribution of LSS, we created a simulated catalog of proper motions from Gaia DR1 (Lindegren et al. 2016). We cross-matched the DR1 catalog with the third release of the Large Quasar Astrometric Catalog (LQAC3; Souchay et al. 2009, 2015) to select a large sample of extragalactic objects and to obtain redshifts. Using the pyGaia ${ }^{3}$ package, we gave each quasar a predicted Gaia end-of-mission proper motion uncertainty based on its ecliptic angle and optical $G$-band magnitude. Then, we gave each quasar a proper motion randomly drawn from the distribution of predicted proper motion uncertainties in order to create a null proper motion noisy data set for

\footnotetext{
${ }^{3}$ https://pypi.python.org/pypi/PyGaia
} 
signal recovery simulations. Our total catalog roughly follows the Sloan Digital Sky Survey (SDSS; York et al. 2000) footprint with additional sparse coverage across the entire sky and contains 189,562 objects with an average proper motion uncertainty of $\sim 210 \mu$ as $\mathrm{yr}^{-1}$.

We found 236,218 pairs with comoving separations < 50 Mpc. For each pair with a separation $<100 \mathrm{Mpc}(610,841$ pairs), we assumed that the pair was at the average comoving distance of the pair constituents and added the expected correlation statistic for equidistant pairs predicted from the matter power spectrum to the correlation statistic calculated from the pair's random noise-consistent proper motions. Then we attempted to recover the injected signal to predict whether Gaia proper motions will be able to detect the transverse peculiar velocities induced by the mass distribution of LSS for close-separation pairs. Note that adding two correlation statistics rather than adding two velocities and then calculating the correlation statistic underestimates the total injected signal. However, it is non-trivial to recover predicted velocities from the injected correlation statistic. Therefore, we use the approximation of added correlation statistics, which is sufficient for this initial estimate. Additionally, the assumption of equidistant pairs overestimates the correlation statistic compared to a full calculation of the statistic for randomly oriented pairs (Darling \& Truebenbach 2018 submitted). But, again, this approximation of sufficient for this initial estimate.

Figure 5 shows the binned correlation statistic for our catalog of simulated Gaia proper motions. The blue stars show the bin averages for the catalog with the expected correlation statistic added to the no-signal statistic calculated from the random noise-consistent proper motions, while the black dots show the bin averages for the catalog with no signal added. The red boxes show the $1 \sigma$ distribution of expected correlation statistics for all pairs in each bin. We performed the same pairs search and $\xi_{i}$ binning for both Gaia catalogs as we did for our proper motion catalog. However, because of the large sample size, we did not include a bootstrap. Instead, the plotted bin values and uncertainties are the maximum likelihood solutions for the bin averages and the $68 \%$ confidence intervals of the probability distributions. Table 2 lists the bin averages and uncertainties, along with several additional bin statistics, while Figure 6 shows the catalog's distribution of pair angular separation. Both the table and figure show that all four bins are dominated by pairs with angular separations less than $10^{\circ}$.

The Gaia catalog simulated proper motions demonstrate that even a subset of 189,562 Gaia extragalactic proper motions will be able to detect a deviation from pure Hubble expansion for close-separation pairs. The first bin with comoving separations $<25 \mathrm{Mpc}$ is a $30 \sigma$ detection and is in good agreement with the input signal. Given the approximations made when calculating the correlation statistic, this significance is likely overoptimistic. However, it demonstrates that a significant detection will be possible with Gaia. Although Gaia proper motions will be much less precise than VLBI proper motions, the two orders of magnitude increase in the number of available extragalactic proper motions will allow a significant detection of the expected transverse peculiar velocity two-point correlation function at physical separations $<25 \mathrm{Mpc}$. The shape and magnitude of the measured correlation will allow a means to test the shape of the theoretical mass power spectrum that is independent of any distance ladder.

There are several additional sources of proper motion uncertainty for Gaia extragalactic objects that are not accounted for in our catalog. Intrinsic active galactic nuclei (AGN) variability can cause an apparent proper motion of up to a few mas over the variability timescale for nearby galaxies (Popović et al. 2012). Microlensing can also cause an apparent centroid location shift as large as tens of $\mu$ as for stellar mass lensing objects (Treyer \& Wambsganss 2004) and a few mas for lensing stellar clusters (Popović \& Simić 2013). Both AGN variability and microlensing will add uncorrelated proper motion noise to Gaia proper motions. However, the expected signal due to the mass distribution of LSS is a correlated, relative proper motion. Therefore, although these effects will add to the overall noise of Gaia proper motions, they will have a less significant effect on the noise of our measured correlation statistic.

\section{CONCLUSIONS}

In this paper, we measured the relative proper motion of all pairs of objects from the VLBA Extragalactic Proper Motion Catalog (Truebenbach \& Darling 2017) with comoving separations less than $1500 \mathrm{Mpc}$. We found that largeseparation pairs with separations $>200 \mathrm{Mpc}$ are consistent with the null signal expected for Hubble expansion to

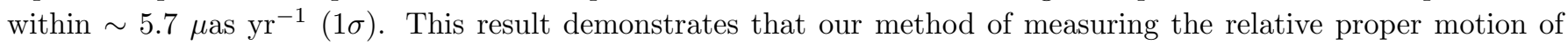
pairs is able to extract small proper motion signals to high precision despite the large scatter of individual pairs due to the intrinsic proper motions from jets.

We also found that we have too few close-separation pairs $(<100 \mathrm{Mpc})$ to statistically detect the expected convergence predicted by the peculiar transverse velocity two-point correlation function. We found a $3 \sigma$ limit on the 


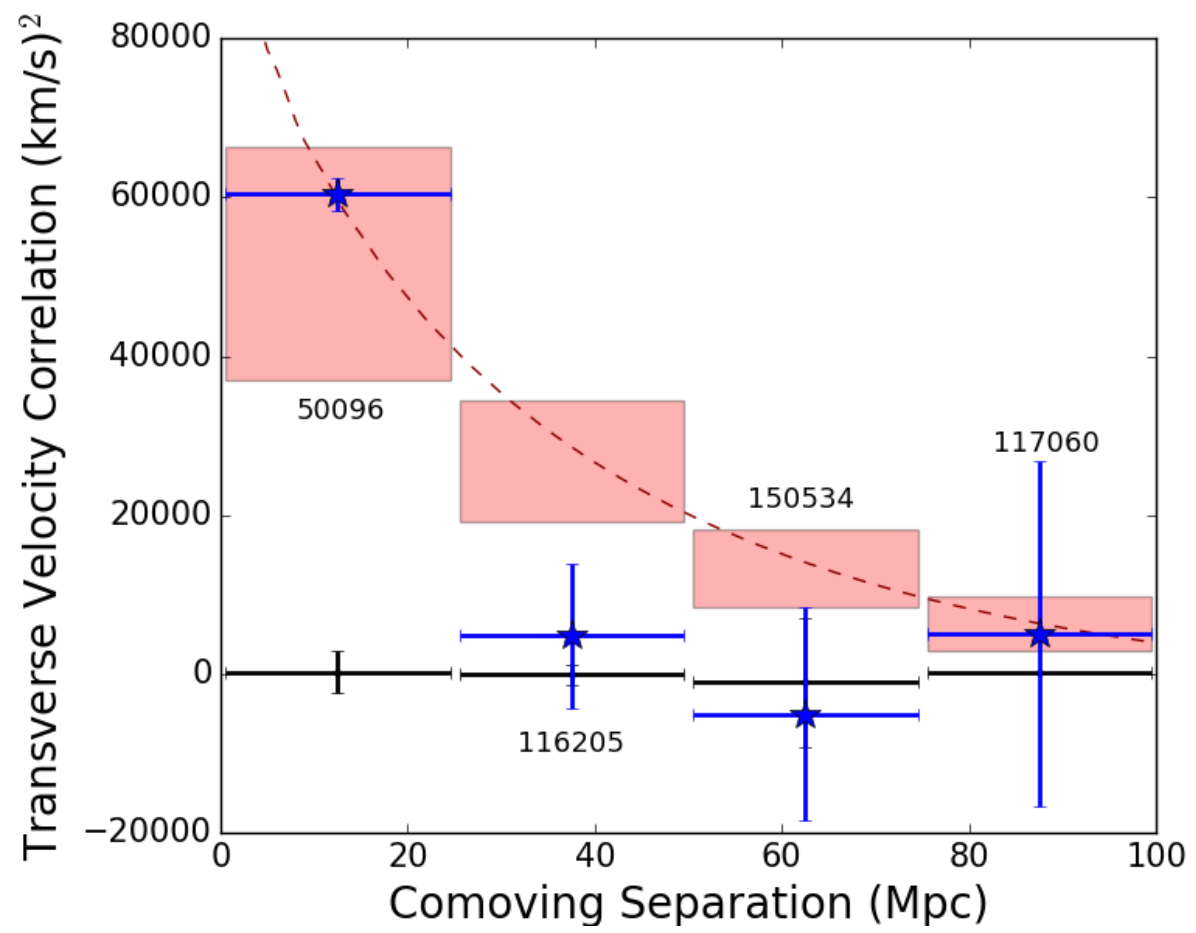

Figure 5. The transverse peculiar velocity two-point correlation statistic (Eqn A7) for the Gaia catalog simulated proper motions. The blue stars show the bin averages for the catalog with the expected correlation statistic added to the no-signal statistic calculated from the random noise-consistent proper motions, while the black dots show the bin averages for the catalog with no signal added. The error bars on these points show the $68 \%$ confidence intervals of the probability distributions of the bin averages. The red boxes show the $1 \sigma$ distribution of expected correlation statistics for all pairs in each bin, while the red dashed line shows the expected correlation statistic from the theoretical mass power spectrum for pairs with angular separations of $5^{\circ}$. The number of pairs per bin is listed above or below each blue star.

Table 2. Gaia Catalog Simulated Proper Motion Statistics

\begin{tabular}{cccccc}
\hline \hline $\begin{array}{c}\text { Comoving Separation } \\
(\mathrm{Mpc})\end{array}$ & $\langle z\rangle$ & $\begin{array}{c}\text { Recovered }\left\langle\xi_{i}\right\rangle \\
\left(\mathrm{km} \mathrm{s}^{-1}\right)^{2}\end{array}$ & $\begin{array}{c}\text { Expected }\left\langle\xi_{i}\right\rangle \\
\left(\mathrm{km} \mathrm{s}^{-1}\right)^{2}\end{array}$ & $\begin{array}{c}\langle\theta\rangle \\
(\mathrm{deg})\end{array}$ & $\mathrm{N}$ \\
\hline $0-25$ & 0.66 & $6.0(0.2) \times 10^{4}$ & $5.2(1.5) \times 10^{4}$ & 5.2 & 50096 \\
$25-50$ & 0.72 & $0.5(0.9) \times 10^{4}$ & $2.6(0.8) \times 10^{4}$ & 7.0 & 116205 \\
$50-75$ & 0.75 & $-0.5(1.3) \times 10^{4}$ & $1.3(0.5) \times 10^{4}$ & 7.7 & 150534 \\
$75-100$ & 0.77 & $0.5(2.2) \times 10^{4}$ & $0.6(0.3) \times 10^{4}$ & 9.4 & 117060 \\
\hline
\end{tabular}

Note-Columns from left to right: Pair comoving separation, average redshift of pairs within separation range, recovered average transverse peculiar velocity twopoint correlation statistic and $1 \sigma$ uncertainties in parentheses, expected average correlation statistic based on pair physical and angular separation with the expected distribution standard deviation in parentheses, average pair angular separation, and number of pairs within separation range. 


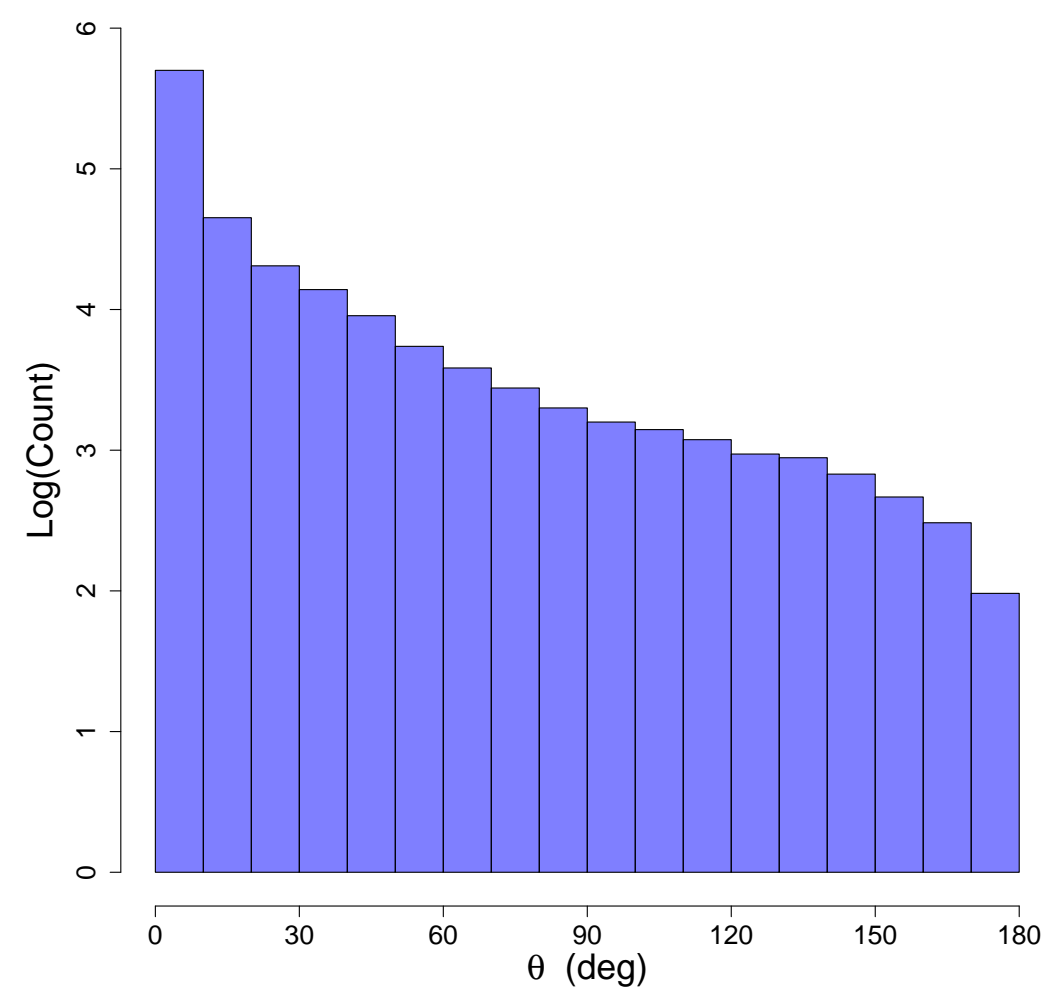

Figure 6. Histogram showing the distribution of pair angular separations in the Gaia catalog with simulated proper motions.

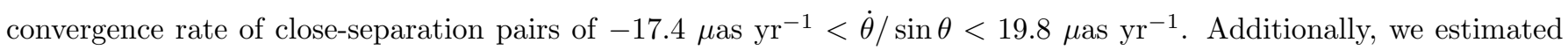
that we would need $\sim 30$ more uncorrelated pairs with separations $<50 \mathrm{Mpc}$ to make a $3 \sigma$ detection of this effect.

There are several available avenues for expanding our sample of close-separation pairs. The best source for additional extragalactic proper motions is very long baseline interferometry because of its uniquely high angular resolution. From the uncertainties in Figure 4, we predict that we would need $\sim 30$ additional pairs with comoving separations $<50$ $\mathrm{Mpc}$ to measure the expected signal from the mass distribution of LSS. However, in order to achieve the proper motion precisions in our catalog, astrometry was obtained for all quasars more than three times over at least a 10 year timespan. Therefore, addition of new close-separation pairs would be a long-term project that would require significant VLBI use.

The Next Generation Very Large Array (ngVLA) provides another avenue for measuring new extragalactic proper motions. The ngVLA will provide ten times the effective collecting area and ten times longer baselines (300 km) than the current Karl Jansky Very Large Array (JVLA; Carilli et al. 2015). The larger collecting area will enable faster astrometry of fainter sources than is currently possible with the VLBA. In one hour of observation time, the ngVLA will detect objects with flux densities of a few tenths of a mJy or brighter (Butler et al. 2018).

If additional ngVLA antennae are installed at VLBA sites, the collecting power of the ngVLA could be combined with the astrometric precision of the VLBA to quickly create a large sample of close-separation pairs. For example, if 10,000 objects were monitored on a yearly basis to obtain $10 \mu \mathrm{as} \mathrm{yr}^{-1}$ astrometry per object, this would enable global

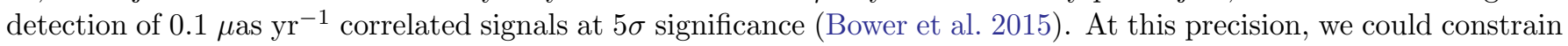
isotropy to $0.1 \%$ of $H_{0}$ (Bower et al. 2015). If objects are selected to be in close-separation pairs, a new proper motion catalog with the ngVLA and VLBA would contain more than enough objects to detect the mass distribution of LSS; we predict that we need only $\sim 30$ pairs with separations $<50 \mathrm{Mpc}$ for a $3 \sigma$ detection of this effect (Section 5).

However, it is uncertain whether the ngVLA will include long baselines $>1000 \mathrm{~km}$. For observations at $3.6 \mathrm{~cm}$ with a maximum baseline of $1000 \mathrm{~km}$, the ngVLA will achieve a spatial resolution of $\sim 7$ mas. If objects are observed

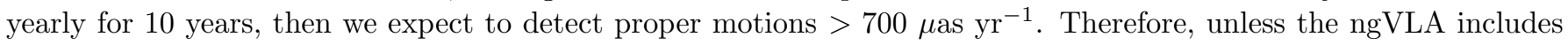


baselines on order of VLBA baselines or can be used in conjunction with existing VLBI networks, the ngVLA will not be an effective tool to expand our sample of close-separation pairs.

With the limitations of current VLBA catalogs and ngVLA baselines, Gaia proper motions remain the best avenue for increasing our sample of close-separation pairs. As discussed in Section 5, Gaia will produce $\sim 10^{6}$ new extragalactic proper motions (Robin et al. 2012) with astrometric precisions of 1.69 mas (Lindegren et al. 2016). Although

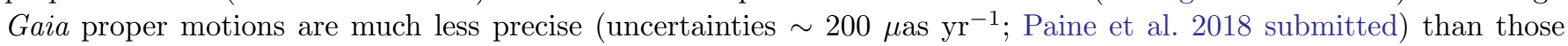

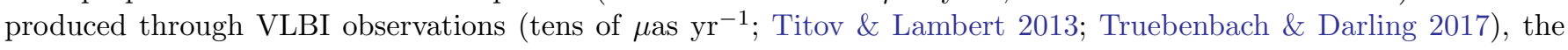
large number of additional proper motions will allow a statistical detection of correlated signals. Additionally, optical proper motions typically have a smaller contribution from intrinsic proper motions than those measured in the radio because the optical source more frequently traces the galaxy core, rather than tracing a jet driven by the active galactic nucleus (Darling et al. 2018 submitted).

In Section 5, we examined a subset of the Gaia DR1 catalog with 610,841 simulated extragalactic proper motions to estimate how well Gaia end-of-mission proper motions will be able to detect the relative velocity of close-separation pairs caused by the mass distribution of LSS. We found that the subset is able to significantly detect the predicted correlation of close-separation pairs. Therefore, although our current sample of close-separation pairs is too small to statistically detect the net convergence of gravitationally-interacting pairs, Gaia proper motions will be able to make a highly significant detection of the mass distribution of LSS on small scales. Comparison of the measured peculiar transverse two-point correlation statistic to that predicted by the theoretical matter power spectrum will allow a means to test the cosmological principles that contribute to the shape and magnitude of the power spectrum without a reliance on precise distance measurements.

\section{ACKNOWLEDGMENTS}

The authors thank David Gordon (NASA Goddard Space Flight Center), Nils Halverson, David Brain, Julie Comerford, and Alysia Marino (all University of Colorado Boulder) for helpful discussions. The authors acknowledge support from the NSF grant AST-1411605 and the NASA grant 14-ATP14-0086. All catalogue comparisons were performed using the tabular manipulation tool STILTS (Taylor 2006). This research has made use of NASA Goddard Space Flight Center's VLBI source time series 2017a solution, prepared by David Gordon. This research has made use of the NASA/IPAC Extragalactic Database (NED) which is operated by the Jet Propulsion Laboratory, California Institute of Technology, under contract with the National Aeronautics and Space Administration.

Software: LMFIT (Newville et al. 2014), STILTS (Taylor 2006) 


\section{APPENDIX}

\section{A. TRANSVERSE PECULIAR VELOCITY TWO-POINT CORRELATION STATISTIC}

For two objects in flat space at locations $\left(\alpha_{1}, \delta_{1}, x_{1}\right)$ and $\left(\alpha_{2}, \delta_{2}, x_{2}\right)$ in spherical coordinates, the vector connecting the two objects, converted to Cartesian coordinates, is

$$
\vec{x}=\left(\begin{array}{c}
D_{1} \cos \alpha_{1} \cos \delta_{1}-D_{2} \cos \alpha_{2} \cos \delta_{2} \\
D_{1} \sin \alpha_{1} \cos \delta_{1}-D_{2} \sin \alpha_{2} \cos \delta_{2} \\
D_{1} \sin \delta_{1}-D_{2} \sin \delta_{2}
\end{array}\right),
$$

where $D_{1}$ and $D_{2}$ are the proper distances of the two objects.

The transverse velocity of each object, in equatorial coordinates, is

$$
\vec{v}_{\perp}=D\left(\mu_{\alpha} \hat{e}_{\alpha}+\mu_{\delta} \hat{e}_{\delta}\right)
$$

where $\hat{e}_{\alpha}$ and $\hat{e}_{\delta}$ are the unit vectors along the right ascension and declination axes and $\mu_{\alpha}$ and $\mu_{\delta}$ are the object's proper motion in right ascension and declination. Using the conversion of $\hat{e}_{\alpha}$ and $\hat{e}_{\delta}$ to Cartesian coordinates from Mignard \& Klioner (2012) (Eqns 5 and 6),

$$
\vec{v}_{\perp}=D\left(\begin{array}{c}
-\mu_{\alpha} \sin \alpha-\mu_{\delta} \cos \alpha \sin \delta \\
\mu_{\alpha} \cos \alpha-\mu_{\delta} \sin \alpha \sin \delta \\
\mu_{\delta} \cos \delta
\end{array}\right),
$$

with associated uncertainty

$$
\sigma_{\vec{v}_{\perp}}^{2}=D^{2}\left(\begin{array}{c}
\sigma_{\mu_{\alpha}}^{2} \sin ^{2} \alpha+\sigma_{\mu_{\delta}}^{2} \cos ^{2} \alpha \sin ^{2} \delta \\
\sigma_{\mu_{\alpha}}^{2} \cos ^{2} \alpha+\sigma_{\mu_{\delta}}^{2} \sin ^{2} \alpha \sin ^{2} \delta \\
\sigma_{\mu_{\delta}}^{2} \cos ^{2} \delta
\end{array}\right) .
$$

We assume that the proper motion uncertainties are significantly larger than those in $\vec{x}, \alpha, \delta$, and $D$.

For each object, we compute $\vec{v}_{\perp} \cdot \hat{x}$ through a normal Cartesian dot product -

$$
\vec{v}_{\perp} \cdot \hat{x}=\frac{1}{|\vec{x}|}\left(v_{\perp, x} x_{x}+v_{\perp, y} x_{y}+v_{\perp, z} x_{z}\right) .
$$

The uncertainty for the dot product is

$$
\sigma_{\vec{v}_{\perp} \cdot \hat{x}}^{2}=\frac{1}{|\vec{x}|^{2}}\left(\sigma_{v_{\perp, x}}^{2} x_{x}^{2}+\sigma_{v_{\perp, y}}^{2} x_{y}^{2}+\sigma_{v_{\perp, z}}^{2} x_{z}^{2}\right),
$$

assuming negligible uncertainty in $\vec{x}$. Therefore, the correlation statistic for two individual objects is

$$
\xi_{i}=\left(\vec{v}_{\perp, 1} \cdot \hat{x}\right)\left(\vec{v}_{\perp, 2} \cdot \hat{x}\right),
$$

with associated uncertainty

$$
\sigma_{\xi_{i}}^{2}=\xi_{i}^{2}\left(\frac{\sigma_{\vec{v}_{\perp, 1} \cdot \hat{x}}^{2}}{\left(\vec{v}_{\perp, 1} \cdot \hat{x}\right)^{2}}+\frac{\sigma_{\vec{v}_{\perp, 2} \cdot \hat{x}}^{2}}{\left(\vec{v}_{\perp, 2} \cdot \hat{x}\right)^{2}}\right) .
$$

\section{REFERENCES}

Bardeen, J. M., Bond, J. R., Kaiser, N., \& Szalay, A. S. 1986, ApJ, 304, 15
Bastian, U. 1995, in ESA Special Publication, Vol. 379, Future Possibilities for bstrometry in Space, ed. M. A. C. Perryman \& F. van Leeuwen, 99 
Blakeslee, J. P., Ajhar, E. A., \& Tonry, J. L. 1999, in Astrophysics and Space Science Library, Vol. 237, Post-Hipparcos Cosmic Candles, ed. A. Heck \& F. Caputo, 181

Blumenthal, G. R., Faber, S. M., Primack, J. R., \& Rees, M. J. 1984, Nature, 311, 517

Bower, G. C., Demorest, P., Braatz, J., et al. 2015, ArXiv e-prints.

Bridle, A. H., \& Perley, R. A. 1984, ARA\&A, 22, 319

Butler, B., Grammer, W., Selina, R., Murphy, E. J., \& Carilli, C. 2018, in American Astronomical Society Meeting Abstracts, Vol. 231, American Astronomical Society Meeting Abstracts, 342.09

Carilli, C. L., McKinnon, M., Ott, J., et al. 2015, ArXiv e-prints.

Coles, P. 1993, MNRAS, 262, 1065

Colgate, S. A. 1979, ApJ, 232, 404

Courtois, H. M., Hoffman, Y., Tully, R. B., \& Gottlöber, S. 2012, ApJ, 744, 43

Darling, J. 2013, ApJL, 777, L21

Darling, J., \& Truebenbach, A. E. 2018 submitted, Connecting Proper Motions to the Matter Power Spectrum: Theory

Darling, J., Truebenbach, A. E., \& Paine, J. 2018 submitted, ApJ

de Lapparent, V., Geller, M. J., \& Huchra, J. P. 1986, ApJL, 302, L1

Dekel, A., Bertschinger, E., Yahil, A., et al. 1993, ApJ, 412, 1

Doumler, T., Courtois, H., Gottlöber, S., \& Hoffman, Y. 2013

Eisenstein, D. J., \& Hu, W. 1999, ApJ, 511, 5

Eisenstein, D. J., Zehavi, I., Hogg, D. W., et al. 2005, ApJ, 633

Eubanks, T. M., Matsakis, D. N., Josties, F. J., et al. 1995, in IAU Symposium, Vol. 166, Astronomical and Astrophysical Objectives of Sub-Milliarcsecond Optical Astrometry, ed. E. Hog \& P. K. Seidelmann, 283

Faber, S. M., \& Jackson, R. E. 1976, ApJ, 204, 668

Fanselow, J. L. 1983, Observation Model and parameter partial for the JPL VLBI parameter Estimation Software "MASTERFIT-V1.0", Tech. rep.

Fey, A. L., Eubanks, M., \& Kingham, K. A. 1997, AJ, 114, 2284

Fry, J. N. 1996, ApJL, 461, L65

Gaia Collaboration, Brown, A. G. A., Vallenari, A., et al. 2018, ArXiv e-prints.

Gaia Collaboration, Prusti, T., de Bruijne, J. H. J., et al. 2016, A\&A, 595, A1
Gott, III, J. R., Jurić, M., Schlegel, D., et al. 2005, ApJ, 624,463

Gwinn, C. R., Eubanks, T. M., Pyne, T., Birkinshaw, M., \& Matsakis, D. N. 1997, ApJ, 485, 87

Hanes, D. A. 1977, MmRAS, 84, 45

Harris, W. E., \& Racine, R. 1979, ARA\&A, 17, 241

Hu, W., Spergel, D. N., \& White, M. 1997, PhRvD, 55, 3288

Hu, W., \& White, M. 1996a, Physical Review Letters, 77, 1687

Jensen, J. B., Tonry, J. L., \& Luppino, G. A. 1998, ApJ, 505,111

Kaiser, N. 1984, ApJL, 284, L9

Kitaura, F.-S., Erdoğdu, P., Nuza, S. E., et al. 2012, MNRAS, 427, L35

Kopeikin, S. M., \& Makarov, V. V. 2006, AJ, 131, 1471

Kovalevsky, J. 2003, A\&A, 404, 743

Lindegren, L., Lammers, U., Bastian, U., et al. 2016, A\&A, 595, A4

Mei, S., Blakeslee, J. P., Tonry, J. L., et al. 2005, ApJS, 156,113

Mignard, F. 2002, in EAS Publications Series, Vol. 2, EAS Publications Series, ed. O. Bienayme \& C. Turon, 327-339

Mignard, F., \& Klioner, S. 2012, A\&A, 547, A59

Moodley, K., Bucher, M., Dunkley, J., Ferreira, P. G., \& Skordis, C. 2004, PhRvD, 70, 103520

Newville, M., Stensitzki, T., Allen, D. B., \& Ingargiola, A. 2014, LMFIT: Non-Linear Least-Square Minimization and Curve-Fitting for Python

Nusser, A., Branchini, E., \& Davis, M. 2012, ApJ, 755, 58

Paine, J., Darling, J., \& Truebenbach, A. E. 2018 submitted, ApJS

Peiris, H. V., Komatsu, E., Verde, L., et al. 2003, ApJS, 148,213

Perlmutter, S., Aldering, G., Goldhaber, G., et al. 1999, ApJ, 517

Planck Collaboration, Adam, R., Ade, P. A. R., et al. 2016a, A\&A, 594, A1

Planck Collaboration, Ade, P. A. R., Aghanim, N., et al. 2016b, A\&A, 594, A13

-. 2016c, A\&A, 594, A20

-. 2016d, A\&A, 594, A14

Popović, L. Č., Jovanović, P., Stalevski, M., et al. 2012, A\&A, 538, A107

Popović, L. Č., \& Simić, S. 2013, MNRAS, 432, 848

Quercellini, C., Quartin, M., \& Amendola, L. 2009, Physical Review Letters, 102, 151302 
Reid, M. J., Menten, K. M., Zheng, X. W., et al. 2009, ApJ, 700, 137

Riess, A. G., Press, W. H., \& Kirshner, R. P. 1996, ApJ, 473, 88

Riess, A. G., Nugent, P. E., Gilliland, R. L., et al. 2001, ApJ, 560, 49

Riess, A. G., Macri, L., Casertano, S., et al. 2011, ApJ, 730, 119

Riess, A. G., Macri, L. M., Hoffmann, S. L., et al. 2016, ApJ, 826, 56

Riess, A. G., Casertano, S., Yuan, W., et al. 2018, ArXiv e-prints.

Robin, A. C., Luri, X., Reylé, C., et al. 2012, A\&A, 543, A100

Sivia, D., \& Skilling, J. 2006, Data analysis: a Bayesian tutorial, Oxford science publications (Oxford University Press).

Souchay, J., Andrei, A. H., Barache, C., et al. 2009, A\&A, 494, 799

-. 2015, A\&A, 583, A75

Sovers, O. J., Fanselow, J. L., \& Jacobs, C. S. 1998, Reviews of Modern Physics, 70, 1393

Strauss, M. A., Davis, M., Yahil, A., \& Huchra, J. P. 1992, ApJ, 385, 421
Taylor, M. B. 2006, in Astronomical Society of the Pacific Conference Series, Vol. 351, Astronomical Data Analysis Software and Systems XV, ed. C. Gabriel, C. Arviset, D. Ponz, \& S. Enrique, 666

Tegmark, M., \& Peebles, P. J. E. 1998, ApJL, 500, L79

Titov, O., \& Lambert, S. 2013, A\&A, 559, A95

Titov, O., Lambert, S. B., \& Gontier, A.-M. 2011, A\&A, 529, A91

Tonry, J., \& Schneider, D. P. 1988, AJ, 96, 807

Tonry, J. L., Ajhar, E. A., \& Luppino, G. A. 1990, AJ, 100, 1416

Treyer, M., \& Wambsganss, J. 2004, A\&A, 416, 19

Truebenbach, A. E., \& Darling, J. 2017, ApJS, 233, 3

Tully, R. B., Courtois, H., Hoffman, Y., \& Pomarède, D. 2014, Nature, 513, 71

Xu, M. H., Wang, G. L., \& Zhao, M. 2012, A\&A, 544, A135

—. 2013, MNRAS, 430, 2633

York, D. G., Adelman, J., Anderson, Jr., J. E., et al. 2000, AJ, 120, 1579

Zhang, B. R., Childress, M. J., Davis, T. M., et al. 2017, MNRAS, 471, 2254 